\title{
NIOBIUM THIN FILM COATING ON A 500-MHZ COPPER CAVITY BY PLASMA DEPOSITION*
}

\author{
H. Wang", G. Wu, H. L. Phillips, R. A. Rimmer, A-M. Valente, A. T. Wu \\ Thomas Jefferson National Accelerator Facility, Newport News, VA 23606, USA
}

\begin{abstract}
A system using an Electron Cyclotron Resonance (ECR) plasma source for the deposition of a thin niobium film inside a copper cavity for superconducting accelerator applications has been designed and is being constructed. The system uses a $500-\mathrm{MHz}$ copper cavity as both substrate and vacuum chamber. The ECR plasma will be created to produce direct niobium ion deposition. The central cylindrical grid is DC biased to control the deposition energy. This paper describes the design of several subcomponents including the vacuum chamber, RF supply, biasing grid and magnet coils. Operational parameters are compared between an operating sample deposition system and this system. Engineering progress toward the first plasma creation will be reported here.
\end{abstract}

\section{INTRODUCTION}

The commonly used superconducting material to produce radio frequency accelerator cavities is high RRR bulk niobium [1]. For a very large accelerator, like the International Linear Collider, a low material cost is one of major concerns. Niobium on copper $(\mathrm{Nb} / \mathrm{Cu})$ thin film technology has been successfully used in several machines [2]. Beyond the material cost, higher thermal stability, less sensitivity to external magnetic fields, potential innovative materials or optimization of BCS resistance at $4.2 \mathrm{~K}$, and possible larger size (lower frequency) of cavity coating are major advantages of the thin film over the bulk niobium technology. Commonly used magnetron sputtering techniques need further improvements [3] to achieve the same performance as bulk $\mathrm{Nb}$ at high gradients. Thin film coating technology combined with surface science analysis for RF superconductivity has become more common in research [4].

Columnar growth in the thin film coating has been commonly seen in magnetron-sputtered niobium on copper substrate. A higher surface adatom mobility [5] could help to suppress this problem. However, a large cavity surface cannot be sufficiently elevated to such a temperature to achieve the needed mobility. The energetic condensation of refractory niobium films achieved through ECR plasma has some advantages over other deposition techniques [6], as ease of deposition energy control, absence of working gas and macroparticles.

A conceptional design to coat a small $(1.5 \mathrm{GHz})$ cavity was first developed [7] based on an existing small sample

\footnotetext{
${ }^{*}$ This work was supported by DOE contract DE-AC05-84ER40150 Modification No. M175, under which the Southeastern Universities Research Association (SURA) operates the Thomas Jefferson National Accelerator Facility.

"E-mail: haipeng@jlab.org
}

coating system. With interest and support from Cornell University for coating a $500 \mathrm{MHz}$ cavity [8], we modified the original design to accomeondate this [9-10]. A new cavity coating lab has been installed at JLab for this collaboration. Rapid progress has been made toward the first milestone of plasma creation inside the cavity.

A parallel program to study the correlation of deposition energy to the surface morphology and chemistry on small samples of $\mathrm{Nb} /$ sappire and $\mathrm{Nb} / \mathrm{Cu}$ is still on going. Those samples were produced by a small independent ECR plasma coating system. Tc and RRR measurements and the surface study results from TEM, SEM, SIMS and 3-D profilometry have been reported [10] and will be updated [11] seperately. The RF surface impedance of small samples are to be measured on a TE011 cavity [12].

\section{CAVITY DEPOSITION SYSTEM DESIGN}

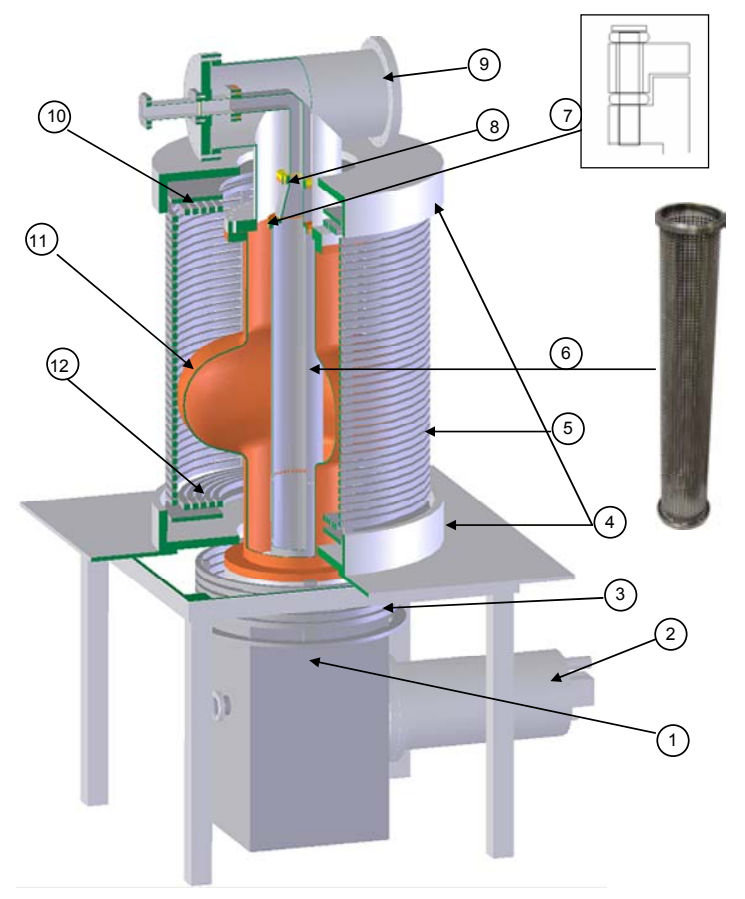

Figure 1: System design of the $\mathrm{Nb}$ thin film equipment coating on a $500 \mathrm{MHz} \mathrm{Cu}$ cavity by plasma deposition. The labels in picture are: (1) $14 \mathrm{~kW}$ rod-fed E-gun; (2) 9000 1/s cryopump system; (3) bucking coil for E-gun; (4) top and bottom iron yokes (outer iron shield is removed for illustration); (5) center coils; (6) $\mathrm{Nb}$ grid tube; (7) bias insulator; (8) WR284 waveguide E-bend and horn to the grid tube; (9) "T" vacuum chamber, (10) top coil; (11) $\mathrm{Cu}$ cavity; (12) bottom coil. 
The generation of plasma inside vacuum requires three essential components: neutral $\mathrm{Nb}$ vapor, $\mathrm{RF}$ power at defined frequency and proper static magnetic field (B) perpendicular to the electric field $(\mathrm{E})$ of the $\mathrm{TE}_{11}$ mode inside the circular waveguide, and the parameters to satisfy the ECR condition. With a variable DC bias voltage between the grid and the substrate, the energy of deposited $\mathrm{Nb}$ ions can be controlled.

\section{Vacuum System}

In the present cavity deposition system (see Figure 1), the cavity itself is part of the vacuum chamber. The vacuum will be generated by a $9000 \mathrm{l} / \mathrm{s}$ cryopump unit coupled with a mechanical roughing pump. In addition, a getter pump will be added on a side port of the E-gun chamber to pump the residual hydrogen, mostly generated by the evaporation of niobium. The cavity flanges, 13.25inch Conflat type, are attached to E-gun chamber at bottom and jointed at the top by a "T" section chamber with a 10-inch flange on each side. The vacuum gauges and an extra getter pump will be installed on the 10-inch flange opposite to the RF window flange. The E-gun is the $\mathrm{Nb}$ vapor source with a $14 \mathrm{~kW}$ heating power and a $\mathrm{Nb}$ rod-feeding mechanism. It can generate $\mathrm{Nb}$ flux at an estimated rate of $130 \mathrm{Angstrom} / \mathrm{sec}$ at the center of the cavity, which is 15 inches away from the heating hearth. This flux rate is comparable to the flux rate in our operating sample coating system. The RF waveguide runs through a window at the 10 -inch flange and is connected by a horn to the axial $\mathrm{Nb}$ grid tube. The grid tube is made of a $\mathrm{Nb}$ sheet perforated with $6.35 \mathrm{~mm}$ holes. The RF field will be confined inside of the tube and only $\mathrm{Nb}$ ions pass through the grid. The grid tube will be insulated from the RF horn and its DC bias voltage will be controlled via a lead wire connected to a feedthrough on the " $\mathrm{T}$ " flange. The use of a niobium grid will reduce the contamination and provide a higher heat tolerance. The RF analysis shows that for a typical $250 \mathrm{~W}$ input power, the grid temperature should be lower than $500^{\circ} \mathrm{C}$. In order to allow the niobium flux to go through and avoid any RF field back to the E-gun, a slit aperture is placed at the bottom of the grid.

\section{RF System}

A $2 \mathrm{~kW}$ narrow band commercial magnetron operated at $2.45 \mathrm{GHz}$ frequency is used to create the plasma. Without plasma ignition, all driving power will be reflected back. An RF circulator and a water cooled load will absorb the full power. All high power transmission uses WR284 series waveguide. The UHV compatible components including the RF window flange, waveguide E-bend and rectangular-to-circular horn are made by custom design. The whole assembly gives a measured value of $-0.55 \mathrm{~dB}$ insertion loss including reflection and attenuation. The iris size of the RF window has been designed so the Voltage of Standing Wave Ratio VSWR = 1.08:1. The iris surface has been polished to withstand a high RF field and to provide a good sitting for a high vacuum indium seal.

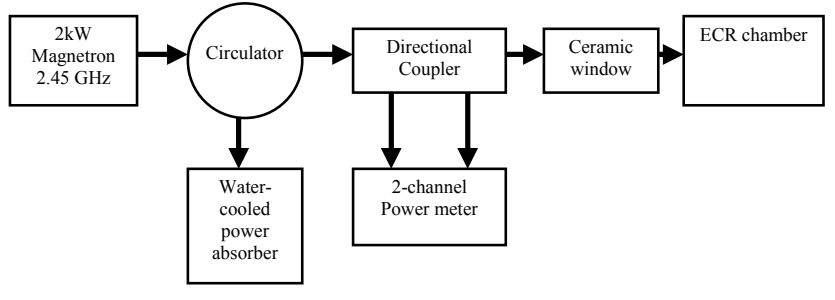

Figure 2: RF system diagram.

The total RF radiation loss through the insulator gap, grid holes and bottom slits has been simulated by HFSS [13], and found to be $11.9 \%$ in standing wave or $3.2 \%$ in traveling wave. The slits on the bottom aperture are designed to follow the RF current pattern in the cross section to minimize the heat generation. When plasma is ignited, the plasma load is quite well matched to the RF source. In the case of a critical power coupling due to the mismatch either before or after the plasma creation, a WR284 waveguide type 3-stub tuner can be used to adjust the coupling. In normal plasma operation, about $500 \mathrm{~W}$ of RF power could be consumed.

\section{Magnet Coil and Power Supply System}

The axial magnetic field is designed to have a uniform field of $875 \pm 7.0$ Gauss in a cylindrical volume of $20 \mathrm{~cm}$ in diameter and $56 \mathrm{~cm}$ in height. The coil magnet system has a $60 \mathrm{~cm}$ internal diameter. It consists of a 35-turn center coil, 5-turn top and bottom coils, top and bottom yokes and a cylindrical shield for magnetic flux return. An additional 5-turn bucking coil will be used to minimize the field distortion in the E-gun region.
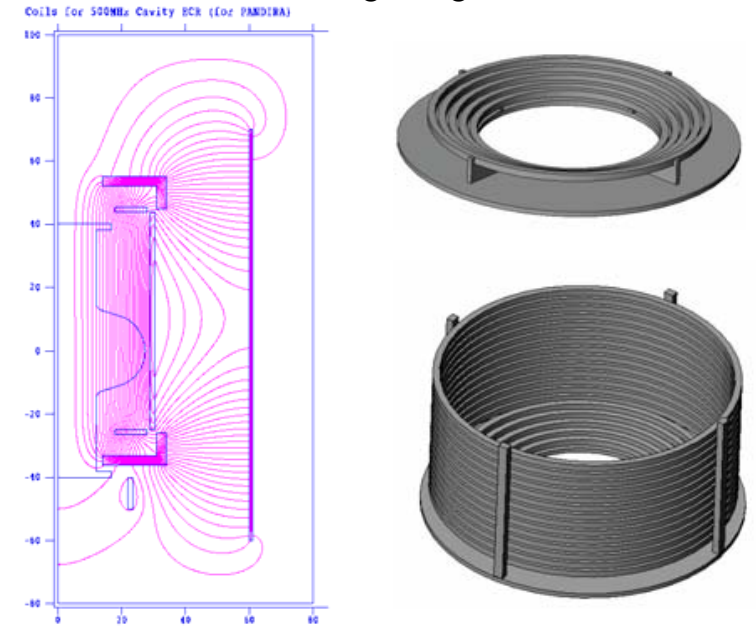

Figure 3: Magnetic field map (left) of the coil-yoke magnet system simulated by Pandria [14] with the uniformity of the ECR requirement at the copper cavity center. The right figures represent the top coil with yoke (top) and center coil (bottom).

The single layer coil design will have a current of 1737 A with a voltage drop of $16.6 \mathrm{~V}$. The heat generated by all the coils is estimated to be about $30 \mathrm{~kW}$. To allow enough heat removal, the copper coil line is hollow to allow cooling water running through. A 2000 A current 
test on two sub coils show that the temperature rise at water outlet is only $7.2^{\circ} \mathrm{F}$ over $80^{\circ} \mathrm{F}$ of water inlet.

Three power supplies are used in parallel to drive the main coils. One Miller welding supply has a 50V/1500A, $100 \%$ duty cycle capacity. The un-regulated current ripple is $0.8 \%$ peak-to-peak in $180 \mathrm{~Hz}$. Two $25 \mathrm{~V} / 250 \mathrm{~A}$ supplies can be run with one of them regulating the ripple further down. The total current will be 1900 A with headroom for regulation.

The second layer coils are going to be made to provide extra ampere-turns to allow for a higher magnetic field if required, or to compensate the fringe field at the cavity beam pipe regions.

\section{WORK IN PROGRESS}

A new thin film lab for cavity deposition has been set up (Figure 4). All utility services have been converted from an old vacuum furnace system including 460V/200A and 208V/400A, 3-phase electrical power, chilled water line and valves, dry nitrogen line, a $250 \mathrm{lb}$ hoist over the coating stand and a sample preparation hood. All power supplies and two regulation schemes have been tested. The copper lines, 85 meters in total, have been wound into sub coils for single layer operation. The cryopump system integrated with the E-gun chamber has been commissioned to achieve a background vacuum of $10^{-8}$ Torr. The testing of the E-gun system and integration of the coil magnet are currently under way. To meet the safety requirements for this $R \& D$ project, we have developed a number of administrative procedures and engineering controls.

We expect the first niobium vapor to be generated during this summer and the first cavity to be coated at the end of this year.

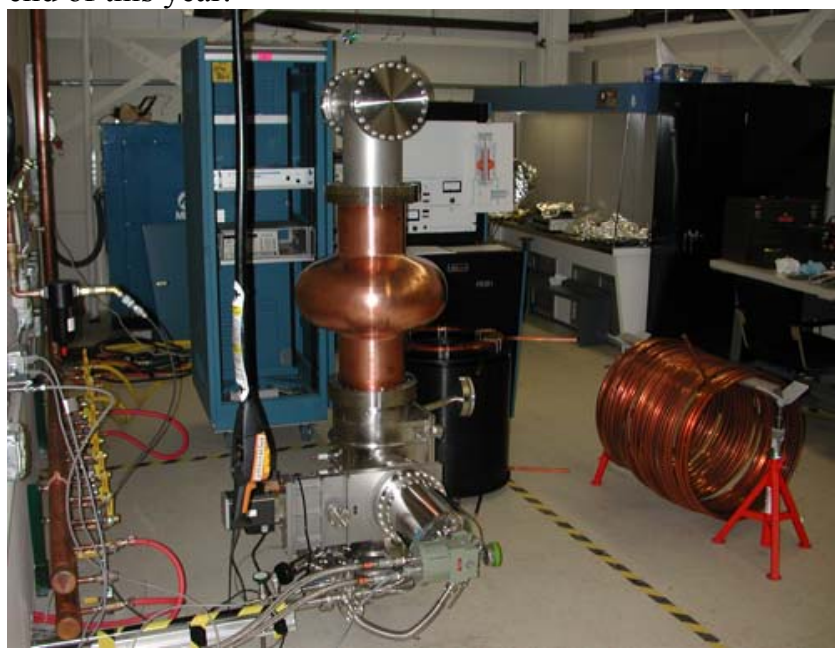

Figure 4: New thin film coating lab is being set up for $500 \mathrm{MHz}$ copper cavity deposition.

\section{ACKNOWLEDGEMENTS}

We are grateful for the technical assistance to R. Bundy, K. Macha, F. Folsom, T. Goodman, R. Manus and many other members of SRF Institute at JLab. Many thanks go to R. Bennett, P. Mutton for their support in facility infrastructure and to J. Musson and P. Powers for their Electrical Engineering service. We also appreciate R. May and P. Hunt for their dedicated ES\&H administration and control in this $R \& D$ project. We acknowledge $H$. Padamsee of Cornell University for his valuable contribution of the $500 \mathrm{MHz}$ cavity and part of the coating equipment.

\section{REFERENCES}

[1] K. Saito, Particle Accelerator Conference 2003 Proc., p462, Portland (2003).

[2] P. Brown, et. al., 9th Workshop on RF Superconductivity, Santa Fe, (1999), edited by B. Rusnak, LANL, Los Alamos, 1.

[3] V. Arbet-Engels, et al., NIM-PR A 463, 1-8 (2001).

[4] G. Wu, et al., Proc. Workshop on Pushing the Limits of RF Superconductivity, APS, ANL, Chicago, Mar. 2005, ANL-05/10.

[5] J. A. Thornton, J. Vac. Sci. Tech. 11, 666 (1974).

[6] G. Wu, et al., J. Vac. Sci. Tech.. A Vol. 21, No. 4, (2003).

[7] G. Wu, JLab tech note, 04-014, (2004).

[8] H. Padamsee, "SRF for Muon Collider", Proc. of $9^{\text {th }}$ Workshop on RF Superconductivity, 587, (1999).

[9] G. Wu, "Niobium Thin Film Cavity Deposition for a 500MHz Copper Cavity”, Proposal II, (2004), JLab tech note $05-050$.

[10] A-M. Valente, et al. "Niobium Thin Film Cavity Deposition by ECR Plasma”, Proc. EPAC2004, p1108-1110.

[11] A.T. Wu, et al. "Surface Study of $\mathrm{Nb} / \mathrm{Cu}$ Films for Cavity Deposition by ECR Plasma”, These Proceedings, TPPT084.

[12] H. Wang et al. "A Sapphire Loaded TE011 Cavity Design for Superconducting Surface Impedance Measurement", presented to Mini-workshop of SRF Institute, JLab Internal, Dec. 2-3, 2003.

[13] HFSS, http://www.ansoft.com.

[14] Poisson/Superfish, http://laacg.lanl.gov/laacg/. 